FORMATION Formation emploi

Revue française de sciences sociales

118 | avril-juin 2012

10 ans de parcours professionnels des jeunes :

l'intérêt des études longitudinales

\title{
Les devenirs socioprofessionnels des sortants sans diplôme: un état des lieux dix ans après la sortie du système éducatif (1998-2008)
}

The social trajectories of unqualified youth: An overview ten years after the school drop-out (1998-2008)

Der sozioprofessionelle Werdegang von Abgängern ohne Abschlusszeugnis: Eine Bestandsaufnahme zehn Jahre nach Verlassen des Systems (1998-2008)

El devenir socioprofesional de los que dejan el sistema educativo sin diploma:

Un estado de la situación diez años después de la salida del sistema educativo

(1998-2008)

Jean-Paul Gehin et Ugo Palheta

\section{(2) OpenEdition}

Journals

Édition électronique

URL : http://journals.openedition.org/formationemploi/3577

DOI : $10.4000 /$ formationemploi.3577

ISSN : 2107-0946

Éditeur

La Documentation française

Édition imprimée

Date de publication : 30 juin 2012

Pagination : 15-35

ISSN : 0759-6340

\section{Référence électronique}

Jean-Paul Gehin et Ugo Palheta, « Les devenirs socioprofessionnels des sortants sans diplôme: un état des lieux dix ans après la sortie du système éducatif (1998-2008) », Formation emploi [En ligne], 118 | avril-juin 2012, mis en ligne le 30 août 2012, consulté le 30 octobre 2020. URL : http://

journals.openedition.org/formationemploi/3577 ; DOI : https://doi.org/10.4000/formationemploi.3577

(c) Tous droits réservés 


\title{
Les devenirs socioprofessionnels des sortants sans diplôme :
}

\section{Un état des lieux dix ans après la sortie du système éducatif (1998-2008)}

\author{
Jean-Paul GEHIN \\ Maître de conférences en sociologie à l'université de Poitiers, chercheur au GRESCO \\ (Groupe de recherche et d'études sociologiques du Centre Ouest)
}

Ugo PALHETA

Docteur en sociologie, chercheur postdoctoral au GRESCO (Groupe de recherche et d'études sociologiques du Centre Ouest) et chercheur associé à l'OSC

(Observatoire sociologique du changement)

Résumé

Les devenirs socioprofessionnels des sortants sans diplôme : Un état des lieux dix ans après la sortie du système éducatif (1998-2008)

Nous présentons ici un état des lieux de l'insertion professionnelle des sortants sans diplôme, à partir de l'enquête à 10 ans du Céreq, auprès de la "Génération 1998 ". Moins nombreux qu'auparavant, ils sont en compétition avec des jeunes diplômés pour les emplois d'exécution. Nous décrivons la diversité de leurs devenirs socioprofessionnels et quantifions l'impact de certains facteurs sur ces devenirs. En effet, à niveau de sortie équivalent, les parcours d'entrée dans la vie professionnelle sont très dissemblables. Enfin, nous éprouvons l'hypothèse selon laquelle, au cours du processus d'insertion professionnelle, l'obtention d'un titre scolaire prime sur le niveau de qualification.

Mots clés : Insertion professionnelle $\bullet$ cheminement professionnel $\bullet$ BNQ -Bas niveau de qualification • origine sociale • enquête génération 1998

Abstract

The social trajectories of unqualified youth. An overview ten years after the school drop-out (1998-2008)

In this article, we give an overview about the transition to work of young people who don't have any diplomas. This study leans on the survey realized by the Céreq (French Centre for Research on Education, Training and Employment), about the 1998 generation, which 
covers the ten first years of the working life of 1998 school leavers. Less numerous than before, they have to compete with youngs graduated to get an access to low-qualified jobs. We describe the diversity of occupational trajectories and quantify the impact of several factors on these trajectories. Indeed, from an equivalent school level, transitions to work are very diversified. Finally, we test the hypothesis that diplomas are more determinant for occupational trajectories than qualification levels.

Key words: Transition from school to work $\bullet$ occupational paths $\bullet$ low level qualifications - social origin $\bullet$ generation 98 survey

Journal of Economic Literature: J 24

Traduction : Auteurs

Par un paradoxe apparent, le meilleur moyen de saisir l'emprise des diplômes sur le devenir socioprofessionnel des jeunes consiste sans doute à rendre compte des trajectoires de celles et ceux qui sortent sans aucun diplôme du système de formation initiale ${ }^{1}$. Au cours des années 80 , l'installation d'un chômage de masse et la seconde explosion scolaire ont en effet rendu presque impérative la possession d'un titre scolaire pour l'obtention rapide d'un emploi stable (Verdier, 2002) ${ }^{2}$. En réponse à l’accroissement brutal de la concurrence sur le marché de l'emploi et à un phénomène de déclassement en cascade (Chauvel, 1998), la situation des sans-diplômes s'est brusquement détériorée. Moins nombreux qu’auparavant, ils se trouvent en compétition avec des jeunes diplômés (en particulier ceux de l'enseignement professionnel court ${ }^{3}$ ) pour l'obtention d'emplois d'exécution dans les services ou l'industrie (Baudelot et Establet, 2000).

Dans cet article, nous nous proposons de dresser un état des lieux de l'insertion professionnelle des sortants sans diplôme sur dix ans, en nous fondant sur l'enquête du Céreq auprès de la "Génération 1998 ". Ce travail s'inscrit dans une recherche collective en cours au GRESCO (Groupe de recherches et d'études sociologiques du Centre Ouest), intitulée "Parcours ", qui vise à affiner la connaissance des itinéraires et des modalités d'entrée dans la vie active des jeunes ayant connu des formes d'exclusion scolaire ou de déscolarisation (Glasman et Oeuvrard, 2004). Des recherches qualitatives sont menées de front et cherchent, avec des méthodologies différentes, à reconstituer les parcours longs d'entrée dans la vie active de jeunes passés par quatre types de structures de remédiation: les dispositifs-relais (Millet et Thin, 2003), les classes SEGPA (Sections d'enseignement général et professionnel adaptées), les classes « DP3 » et « DP6 » (anciennement « techno-

1. On n’inclut pas ici le Brevet des collèges.

2. Dans cet article, on considèrera comme "stable " un emploi en CDI (contrat à durée indéterminée) ou sous statut de fonctionnaire.

3. Nous incluons ici aussi bien les filières scolarisées (en lycée professionnel) que par alternance (ce qu’on appelle l'apprentissage). 
logiques ») $)^{4}$, et les actions spécifiques des politiques régionales de formation/insertion des jeunes dits de "bas niveau de qualification" (Géhin, 2009).

Les premiers résultats de ces travaux confirment l'importance des titres scolaires dans les parcours d'entrée dans la vie active des jeunes enquêtés, mais ils révèlent aussi l'existence d'obstacles non-scolaires. L'absence de diplôme n'explique pas, à elle seule, les cas de disqualification sociale ${ }^{5}$; c'est au contraire le cumul du déficit scolaire et de la faiblesse du capital social détenu par la famille d'origine, conjugué parfois à des formes diverses de discriminations (racistes ou sexistes) qui amène une partie des jeunes à s'inscrire dans des processus de disqualification sur le marché du travail, mais aussi matrimonial, et donc à se trouver l'objet d'une exclusion sociale plus ou moins prononcée.

Pour renforcer et compléter ces approches essentiellement qualitatives, l'exploitation statistique de l'enquête à 10 ans auprès de la "Génération 1998 ", réalisée par le Céreq, s'avère pertinente ; en effet, la saisie de cursus individuels longs permet de mesurer le degré d'intégration (ou de distanciation) au monde du travail, de proposer une description de la diversité des devenirs socioprofessionnels au sein de cette fraction de la jeunesse, et idéalement de quantifier l'impact d'une variété de facteurs sur ces devenirs. Nos travaux qualitatifs montrent en effet qu’à niveau de sortie équivalent du système éducatif se dessinent des parcours d'entrée dans la vie très dissemblables. Dès lors, quelles sont les variables qui influent sur ces destins socioprofessionnels? Autrement dit, sous quelles conditions les sortants sans diplôme parviennent-ils à s'engager dans des processus d'intégration durable au monde du travail ou, au contraire, dans des processus de disqualification sociale ?

Nous avons choisi de travailler sur la catégorie statistique des sortants non diplômés, différente de celle utilisée par l'Éducation nationale ; cette dernière met en effet l'accent sur les sortants sans qualification et considère de fait comme qualifiés les jeunes ayant quitté l'école en dernière année de leur formation mais n’ayant pas obtenu le diplôme la validant. Contrairement à ces approches, nous avons intégré, dans la population étudiée, les jeunes qui composent la catégorie des «qualifiés non diplômés » de niveau 5 (sortants sans diplôme de dernière année de CAP-BEP ${ }^{6}$ ) ou de niveau 4 (sortants sans diplôme de terminale générale ou technologique $\left.{ }^{7}\right)$.

4. Ce sigle renvoie à la vocation préprofessionnelle de ces classes puisqu'il signifie " découverte professionnelle "; le chiffre désignant le nombre d'heures hebdomadaires consacrées à ce module.

5. On utilisera cette notion dans le sens que lui donne Serge Paugam (1991), analysant la pauvreté comme une construction sociale articulant des dimensions matérielles, familiales, culturelles, professionnelles, résidentielles.

6. Certificat d'aptitude professionnelle et Brevet d'études professionnelles.

7. On ne trouve pas de jeunes passés par un baccalauréat professionnel dans cette catégorie dans la mesure où l'entrée en baccalauréat professionnel nécessitait auparavant l'obtention préalable d'un diplôme professionnel de niveau 5 (BEP ou CAP). Ce n'est plus le cas depuis la création récente du «bac pro » en 3 ans, préparé après la $3^{\text {ème }}$. 
Ce choix induit l'hypothèse qu'au cours du processus d'insertion professionnelle, c'est moins la qualification qui prime que le titre scolaire, donc moins le contenu de la formation elle-même que le "signal » envoyé par la certification aux employeurs potentiels (Spence, 1973). Un tel choix est cohérent avec l'évolution des politiques éducatives en France, durant les années 80 . Politiques qui mettent de plus en plus l'accent sur la certification (Beaud, 2002 ; Rose et Teissier, 2006) non seulement en fixant des objectifs chiffrés en termes de diplômes mais aussi en favorisant une augmentation sensible du taux de réussite aux examens, ou encore en multipliant les voies d'accès au diplôme, comme c'est le cas pour la validation des acquis de l'expérience (Neyrat, 2007 ; Gehin et Auras, 2011). Face à la montée en puissance de la certification et du diplôme, qui s'exprime également dans les politiques de recrutement des entreprises (Eymard-Duvernay et Marchal, 1997 ; Gorgeu et Mathieu, 2009), il apparaît pertinent de s'intéresser à la catégorie des «nondiplômés ", plutôt qu’à celle des « non-qualifiés ».

Cet article propose donc une exploration statistique des devenirs socioprofessionnels des sans-diplômes. Bien que souvent évoquée dans le débat public, généralement afin de pointer l'inefficacité persistante du système de formation, cette population demeure méconnue et se trouve souvent réduite, implicitement, à un bloc homogène de dépossédés, alors même que - comme on le verra dans les lignes qui suivent - elle renferme des situations très diverses. On vise donc, dans un premier temps, à quantifier l'impact d'une multiplicité de facteurs sur les processus d'insertion des non-diplômés (origine sociale, sexe, etc.), et, dans un second temps, à interroger la persistance, à moyen et long terme, du signal négatif que constitue l'absence de tout titre scolaire, en mesurant l'influence respective du diplôme et de l'expérience.

\section{Les sans-diplômes: dépossession commune, diversité des devenirs}

L'enquête à 10 ans auprès de la "Génération 1998 ", réalisée par le Céreq, permet de confirmer le rôle déterminant du diplôme sur le marché du travail. Guère plus de la moitié des jeunes sortis sans aucun diplôme en 1998 occupaient, dix ans plus tard, un emploi stable (CDI ou fonctionnaire), contre 74 \% pour l'ensemble de la cohorte. De même, plus d'un jeune sans diplôme sur quatre est sans emploi en 2008, contre 5,2 \% pour les diplômés de niveau « bac +3 et plus » comme pour ceux de niveau « bac +2 », 10,8 \% pour les bacheliers et 12,9 \% pour les titulaires d'un CAP ou d'un BEP. Pour autant, l'absence de diplôme n'équivaut pas mécaniquement à une exclusion du marché de l'emploi. Si les sans-diplômes rencontrent des difficultés importantes au sortir du système éducatif, la majorité d'entre eux finit par s'insérer, quoique sous des statuts plus précaires et avec des niveaux de salaire plus faibles. 


\section{Encadré 1}

\section{Les sans-diplômes dans l'enquête 2008 du Céreq, auprès de la « Génération 1998 »}

Prise dans son ensemble et d'après l'enquête 2008 auprès de la " Génération 1998 », la population des sortants sans diplôme représentait 112976 individus en 1998, soit 15,3\% de la cohorte, mais seulement $11,2 \%$ des individus interrogés en 2008, c'est-à-dire 1224 enquêtés (voir tableau 1 infra). Cette sous-représentation est liée au phénomène d'attrition qui touche particulièrement les sortants les plus précoces du système scolaire, en particulier ceux de SEGPA (sections d'enseignement général et professionnel adaptées) et de collège. II s'avère en effet très difficile de contacter et d'interroger quatre fois de suite sur 10 ans certains d'entre eux, plus touchés que les jeunes de leur génération par des ruptures qui sont souvent à la fois scolaires, sociales et familiales (Millet et Thin, 2005). Couplé au problème de la faiblesse des effectifs (qui concerne aussi les $2^{\text {nde }}-1$ ère), cela nous conduira à analyser avec précaution les données pondérées relatives à ces deux catégories de sortants sans diplôme.

\section{Tableau 1}

\section{Effectifs et pourcentages de non-diplômés}

\begin{tabular}{|l|c|c|c|c|}
\hline $\begin{array}{l}\text { Dernière classe } \\
\text { fréquentée }\end{array}$ & $\begin{array}{c}\text { Effectifs non } \\
\text { pondérés }\end{array}$ & $\begin{array}{c}\text { Effectifs } \\
\text { pondérés }\end{array}$ & $\begin{array}{c}\text { Pourcentage dans la } \\
\text { population totale }\end{array}$ & $\begin{array}{c}\text { Pourcentage dans } \\
\text { la population des } \\
\text { non-diplômés }\end{array}$ \\
\hline SEGPA & 53 & 6702 & 0,9 & $8,9 \%$ \\
\hline Collège & 179 & 22280 & 3 & $7,7 \%$ \\
\hline 1ère année CAP-BEP & 362 & 29610 & 4 & $31,6 \%$ \\
\hline 2ème année (AP-BEP & 409 & 35665 & 4,8 & $26,2 \%$ \\
\hline 2nde et 1ère ${ }^{*}$ ) & 105 & 8719 & 1,2 & $19,7 \%$ \\
\hline Terminale & 116 & 10000 & 1,3 & $5,9 \%$ \\
\hline Total des non-diplômés & $\mathbf{1 2 2 4}$ & $\mathbf{1 1 2} \mathbf{9 7 6}$ & $\mathbf{1 5 , 2}$ & $\mathbf{1 0 0} \%$ \\
\hline
\end{tabular}

Source : Enquête à 10 ans, auprès de la "Génération 1998 », Céreq.

Sigles : CAP : certificat d'aptitude professionnelle ; BEP : brevet d'études professionnelles.

(*) De l'enseignement général et technologique.

De manière attendue, les sorties sans diplôme concernent en priorité les jeunes ayant au moins un parent chômeur ou inactif, et ceux appartenant aux classes populaires : ce risque touchait, en 1998, plus d'un enfant d'ouvriers sur quatre et plus d'un enfant d'employés ou d'artisans, commerçants et patrons d'entreprises de plus de 10 salariés (ACP) sur six, mais seulement un enfant de cadres et professions intellectuelles supérieures (CPIS) sur quinze.

Au sein du groupe des sans-diplômes, on observe des variations sensibles selon la classe de sortie. Ainsi, les jeunes issus des classes populaires qui sortent du système de formation sans diplôme le font très majoritairement après un passage dans l'enseignement professionnel court (c'est-à-dire après avoir fréquenté une classe de CAP ou de BEP). En outre, ils sont moins nombreux que ceux issus des classes intermédiaires et favorisées à être sortis, sans diplôme, des filières générales ou technologiques de l'enseignement secondaire. Ces différences peuvent s'expliquer par la propension des parents de milieux favorisés, à niveau scolaire équivalent, à formuler des vœux d'orientation plus ambitieux que les familles populaires et à éviter les filières professionnelles lors de la procédure d'orientation en fin de $3^{\text {ème }}$ (Palheta, 2012). 
... suite de l'encadré 1

Par ailleurs, la population des sortants sans diplôme est masculine à $61,5 \%$. Elle s'avère mécaniquement plus jeune que la moyenne des sortants de $1998: 52,1 \%$ des non-diplômés avaient ainsi 18 ans ou moins au moment de leur sortie du système éducatif, contre seulement $15,4 \%$ pour l'ensemble de la cohorte. La zone d'habitation a un impact sur le type de titre scolaire obtenu par les jeunes, puisque la proportion de diplômés de niveau « bac +3 et plus » croît quand on va des espaces à dominante rurale aux pôles urbains (de $8,5 \%$ à $25,8 \%$ ); cependant, la proportion de sortants sans diplôme ne varie que marginalement selon cette variable, de $16,7 \%$ dans les premiers à $13,8 \%$ dans les seconds.

Le risque de sortir sans diplôme est plus marqué pour les enfants dont le père est né à l'étranger (sauf pour ceux originaires d'Europe du sud), les inégalités selon l'origine géographique recoupant sans doute en partie l'ancienneté de la migration : $45 \%, 33,1 \%, 31,2 \%$ et $28,2 \%$ des enfants de pères nés en Turquie, en Asie, au Maghreb et en Afrique subsaharienne sortent sans diplôme du système de formation, contre $13,6 \%$ des jeunes dont le père est né en France $\left(^{* *}\right)$.

Enfin, preuve que les sorties sans diplôme sont le produit de difficultés précoces d'apprentissage, une forte corrélation apparaît entre le redoublement à l'école primaire et la probabilité d'une sortie sans diplôme. La moitié des sortants sans diplôme a en effet connu au moins un redoublement à l'école primaire, contre moins du quart pour l'ensemble de la cohorte $(22,5 \%)$. Prégnant pour les sorties au niveau SEGPA et collège, ce poids du redoublement ne concerne pas les sortants sans diplôme de $2^{\text {nde }}$ 1 $^{\text {ere }}$ et terminale, dont la scolarité à l'école primaire - du moins au regard de cet indicateur - apparaît similaire à celle du reste de la cohorte.

$\left.{ }^{(* *}\right)$ Ce résultat concorde avec ceux obtenus par Y. Brinbaum et A. Kieffer (2009), sans qu'il soit aisé d'avancer une hypothèse explicative à cet effet de l'origine migratoire, d'ailleurs variable selon la zone géographique d'origine ; et ce d'autant plus que la littérature sur le sujet souligne le surcroît d'ambition scolaire des familles immigrées (à position sociale contrôlée).

Ainsi, on est assez loin de la représentation d'une minorité d'exclus faisant face à une majorité d'inclus. Les contrats précaires ne touchent pas seulement les sortants sans diplôme, même si ces derniers sont surreprésentés dans ces situations : parmi ceux qui sont en emploi dix ans après leur sortie du système de formation, 10,2 \% sont ainsi en CDD (contrat à durée déterminée), 6,6\% en intérim et 3,9\% sont l'objet de contrats atypiques (contre 6,2 \%, 2,2 \% et 1,7\% dans l'ensemble de la cohorte).

Les inégalités face à l'emploi traversent la population des sortants sans-diplôme : leurs trajectoires d'entrée dans la vie active varient de l'accès rapide à l'emploi stable et durable à l'éloignement prolongé du monde du travail, voire à des formes de disqualification sociale impliquant le cumul de propriétés négatives (chômage de longue durée, incapacité de partir du foyer parental, célibat prolongé, etc.), en passant par toute une gamme de situations intermédiaires. Selon le triple critère retenu ici (emploi ou non, statut d'emploi, niveau de salaire), on observe en effet une grande diversité : les sans-diplômes se répartissent à peu près également dans les quatre catégories suivantes : non-emploi, emploi précaire ou stable à moins de 1000 euros, emploi stable entre 1000 et 1499 euros, emploi stable à 1500 euros et plus, alors que plus de trois quart des « bac +3 et plus » se situent dans l'emploi stable à plus de 1500 euros. 


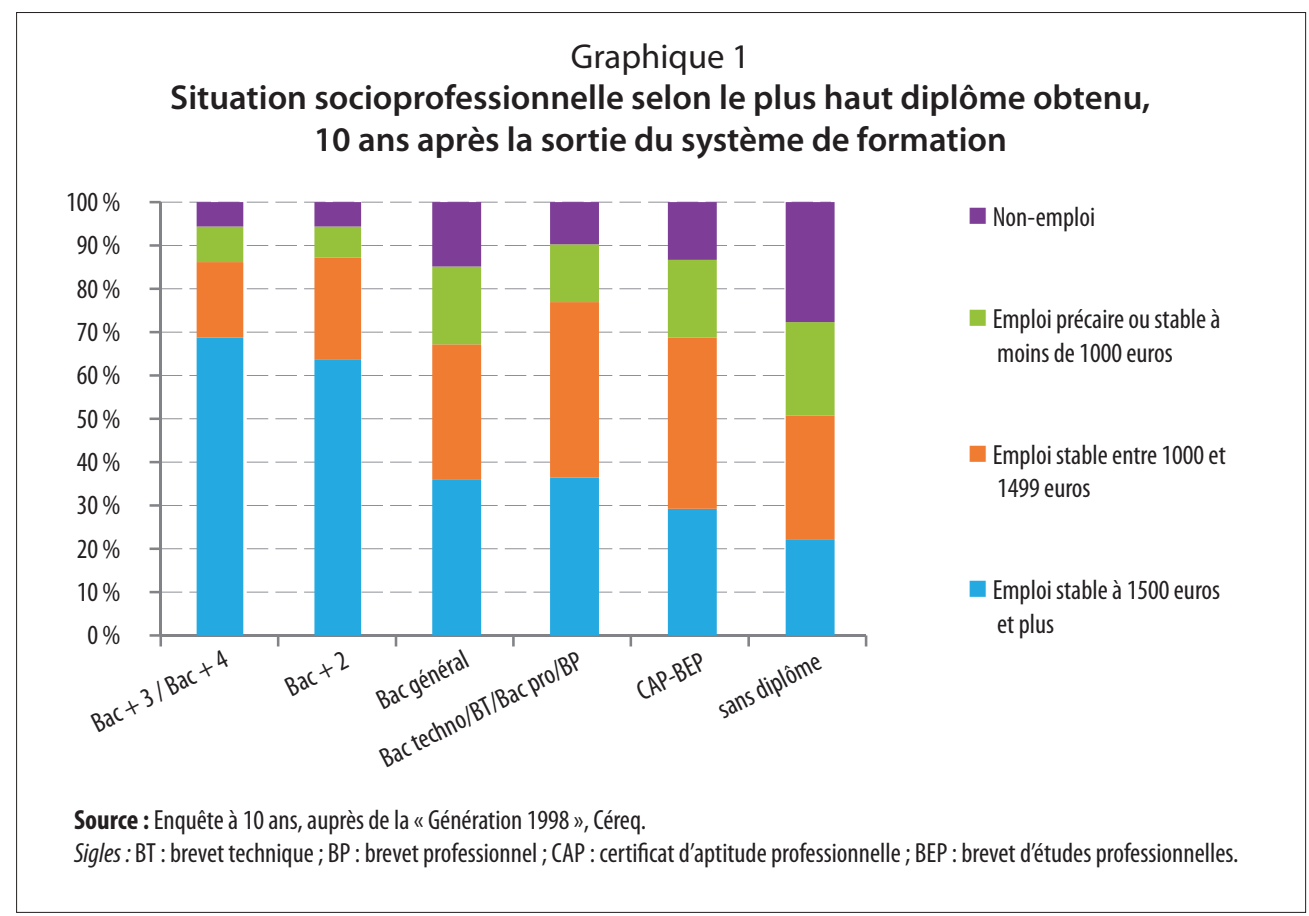

Un tel constat réfute d'emblée aussi bien l'idée selon laquelle l'absence de diplôme interdirait toute intégration au monde du travail que l'affirmation optimiste selon laquelle tous les jeunes, même privés de titre scolaire, finiraient bon an mal an, au bout de quelques années, par se stabiliser dans l'emploi. Plus largement, cela invite à décrire les processus d'insertion professionnelle en refusant tout déterminisme mécanique et en restant attentif aux caractéristiques précises du parcours scolaire comme aux facteurs qui, une fois les jeunes sortis de l'école, différencient les trajectoires d'entrée dans la vie (capital social, pratiques discriminatoires fondées sur le genre ou les caractéristiques ethno-raciales, etc.). Après un examen approfondi, quatre facteurs nous ont paru prédominants : la classe de sortie, l'origine sociale, l'origine migratoire et le sexe.

\subsection{Diversité des devenirs selon la classe de sortie}

Parmi les jeunes non diplômés, le temps moyen d'accès au premier emploi varie d'un peu plus de 8 mois pour les sortants de terminale à environ 20 mois pour les sortants de collège et plus de 32 mois pour les sortants de SEGPA, les sortants de $2^{\text {nde }}-1^{\text {ère }}, 2^{\text {ème }}$ ou $1^{\text {ère }}$ année de CAP-BEP se situant à un niveau très proche (entre 10 et 13 mois).

Ces inégalités perdurent : le taux de chômage et la proportion de jeunes employés dans des conditions précaires, dix ans après leur sortie du système éducatif en 1998, croissent de manière quasi-linéaire quand on va des sans-diplômes sortis après une classe de ter- 


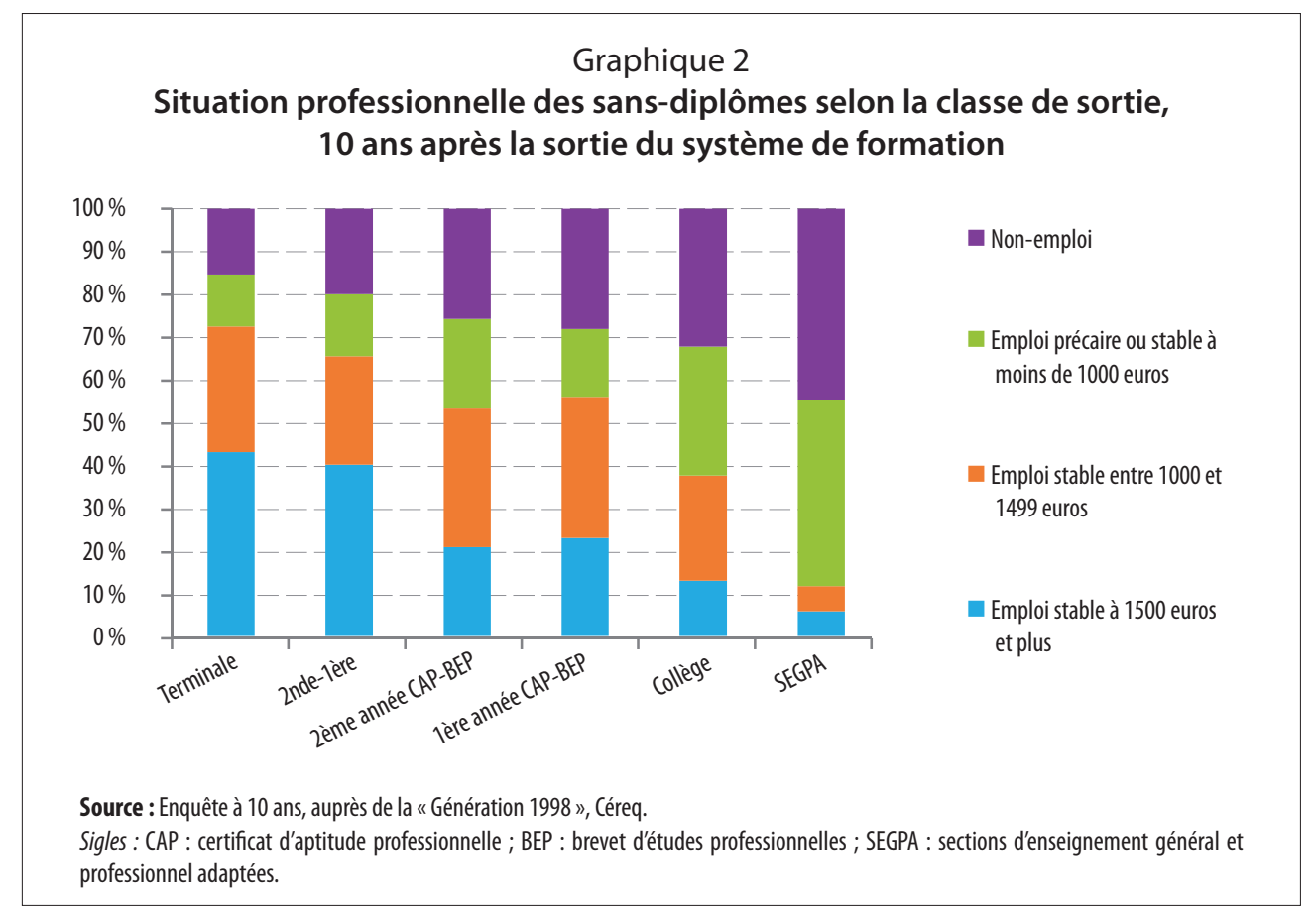

minale (respectivement $9,5 \%$ et $9,9 \%$ ) à ceux qui sont sortis du système de formation après une classe SEGPA (39,2\% et 28,9\%). 70 \% des premiers occupent, en 2008, un emploi stable rémunéré au moins à 1000 euros, ce qui les rapproche des diplômés de niveau CAP-BEP et des bacheliers, alors que c'est le cas de seulement 11,7\% des jeunes sortis après une SEGPA. Sur dix ans, les sortants de terminale auront passé en moyenne moins de 15 mois au chômage contre près de 64 mois, c'est-à-dire plus de cinq ans, pour les sortants de SEGPA, alors que parmi les non-diplômés passés par l'enseignement professionnel - $1^{\text {ère }}$ année comme $2^{\text {ème }}$ année de CAP-BEP - on observe un niveau équivalent (entre 25,5 et 27 mois).

Le graphique 2 illustre ces inégalités devant l'emploi, et particulièrement devant l'emploi stable correctement rémunéré, au sein même de la population des sans-diplômes.

Avec des taux de chômage et d'emplois précaires près de deux fois plus élevés que ceux des jeunes sortis après une classe générale de collège ou une $1^{\text {ère }}$ année de CAP-BEP, les sortants de SEGPA font figure d'exclus durables de l'emploi stable (Zaffran, 2010 ; Palheta, 2012). Sans doute deux logiques se combinent-elles ici : d'un côté, leur faible niveau d'acquisition scolaire rend difficile l'accès à un emploi dans un contexte de chômage de masse et de forte concurrence pour des emplois d'exécution raréfiés ; de l'autre, il est pro- 
bable que le passage par une classe SEGPA produise un effet de signal négatif et accroisse encore les difficultés éprouvées par ces jeunes ${ }^{8}$.

Si les jeunes sortis de terminale et de SEGPA polarisent la population des sans-diplômes, les jeunes passés par une formation professionnelle de niveau CAP-BEP en composent le cœur, sur le plan numérique $(57,8 \%)$ mais surtout parce qu'ils occupent une position intermédiaire entre les sorties précoces (SEGPA et collège) et les sortants sans diplôme de $2^{\text {nde }}, 1^{\text {ère }}$ et terminale. On notera à ce sujet la grande proximité du devenir socioprofessionnel des sortants de première année et d'année terminale de CAP-BEP, deux catégories que les nomenclatures traditionnelles différencient fortement, puisqu'ils sont considérés d'un côté comme "non qualifiés » et de l'autre comme "qualifiés ".

La diversité des devenirs socioprofessionnels selon la classe de sortie se confirme lorsque l'on observe la position socioprofessionnelle occupée par les jeunes en emploi 10 ans après la sortie du système de formation. Si les jeunes sortis après une classe de l'enseignement général ( $2^{\text {nde }}, 1^{\text {ère }}$ ou terminale) sont ouvriers qualifiés aussi souvent que ceux issus de l'enseignement professionnel court, ou même que les jeunes sortis après le collège ou une classe SEGPA, ils sont beaucoup plus rarement ouvriers non-qualifiés et appartiennent fréquemment aux professions intermédiaires, ce que met en évidence le graphique 3.

Lindicateur des salaires obtenus par ceux qui sont en emploi va dans le même sens : alors que les sortants non diplômés du lycée général et technologique se caractérisent par un salaire médian net de 1500 euros, ce niveau s'établit respectivement à 1315 euros pour les sortants non diplômés de l'enseignement professionnel et à 1175 euros pour les sortants de collège ou SEGPA.

\subsection{L'origine sociale joue en amont et en aval de l'insertion}

Au-delà de ses effets sur le cursus scolaire lui-même, l'origine sociale joue un rôle significatif dans le processus d'insertion : les enfants de cadres supérieurs ont ainsi patienté 9 mois en moyenne pour trouver un $1^{\text {er }}$ emploi et ont totalisé 24 mois de chômage sur dix ans, contre 16 et 31 mois pour les enfants d'ouvriers. En 2008, 34,1\% des premiers occupaient un emploi stable rémunéré à plus de 1500 euros, contre $17 \%$ pour les seconds. On notera que les inégalités selon la classe de sortie, dont on a rendu compte plus haut, traduisent sans doute en bonne partie des différences d'origine sociale, puisque ces dernières ont un impact sur la classe de sortie. Linsertion plus favorable des sortants non diplômés de l'enseignement général tient donc aussi bien aux propriétés acquises dans le

8. On notera qu'on ne peut déduire de ces constats statistiques une analyse d'ensemble sur les dispositifs SEGPA dans la mesure où ne sont concernés ici par l'analyse que les jeunes sortis du système de formation après une classe SEGPA, et non l'ensemble des jeunes passés par ce type de dispositif; une bonne partie d'entre eux poursuivant leur cursus dans l'enseignement professionnel, en lycée professionnel ou en apprentissage. 


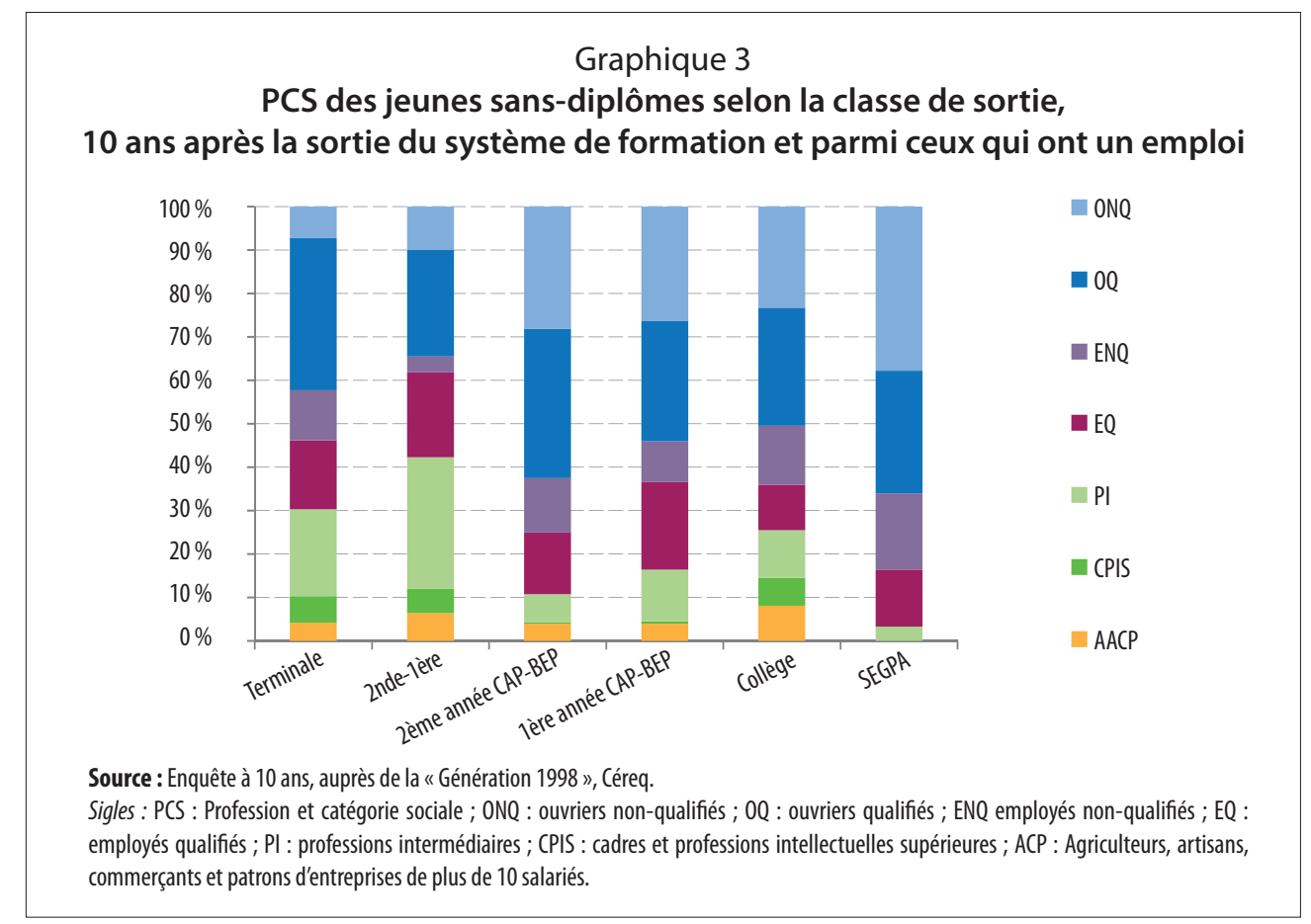

milieu familial (par exemple un capital social monnayable sur le marché du travail) qu’aux caractéristiques propres à leur scolarité, et à leur meilleur niveau d'acquisition scolaire.

En restant prudent en raison des faibles effectifs, il semble que, à classe de sortie identique, les jeunes dont le père appartient aux catégories des "professions intermédiaires " et des " cadres et professions intellectuelles supérieures » soient plus nombreux à occuper un emploi stable rémunéré à plus de 1500 euros. Ainsi, parmi les sortants de classes de CAP-BEP, 30,3\% des jeunes issus des classes intermédiaires et favorisées occupent ce type d'emploi en 2008, contre seulement 16,7 \% des enfants d'ouvriers. Concernant le salaire, il est également difficile de déterminer ce qui tient à la classe de sortie ou au milieu social d'origine ; en effet, le salaire médian des sans-diplômes dont le père est cadre supérieur s'établit à 1500 euros, contre 1400 pour un père faisant partie des professions intermédiaires, 1350 pour les enfants de petits patrons, 1300 pour les enfants d'employés et 1280 pour les enfants d'ouvriers.

Lorigine sociale joue donc en amont et en aval : d'un côté, les jeunes issus des classes intermédiaires et favorisées ont plus de chances, quand ils sont non diplômés, de sortir de l'enseignement général et technologique plutôt que de l'enseignement professionnel, du collège ou de l'enseignement spécialisé ; de l'autre, à niveau de sortie équivalent, ils ont plus de chances d'échapper au chômage et aux segments les plus dévalorisés du salariat. 


\subsection{Une insertion variable selon l'origine migratoire}

L'origine migratoire induit également des effets significatifs sur l'insertion professionnelle des sortants sans diplôme, nos données confirmant les résultats présents dans la littérature sociologique sur la question (Frickey, Murdoch \& Primon, 2004 ; Lainé \& Okba, 2005; Fournier \& Silberman, 2006 ; Meurs, Pailhé \& Simon, 2006).

Elles montrent que les jeunes dont le père est né en Turquie et au Maghreb mettent non seulement plus de temps à trouver un emploi (18 mois pour les premiers et 21 pour les seconds, contre 13 mois pour les jeunes dont le père est né en France), mais pâtissent davantage du chômage sur les dix ans (38 et 44 mois contre 25), et ont plus de chances de se trouver au chômage 10 ans après la sortie du système de formation que les jeunes dont le père est né en France (50,4\% et 45,6\% contre 24,2\%).

De surcroît, lorsqu'ils occupent un emploi, les jeunes d'origine maghrébine sont plus nombreux à pâtir d'un statut précaire $(32,8 \%$ contre $20,2 \%$ des jeunes dont le père est né en France et 18,2 \% pour ceux dont le père est né en Europe du sud). Au total, les jeunes d'origine turque ou maghrébine sortis sans diplôme en 1998 ne sont que $10 \%$ à occuper un emploi stable rémunéré à 1500 euros au moins, dix ans après leur sortie du système de formation, contre $24,8 \%$ des jeunes dont le père est né en France et $26,3 \%$ de ceux originaires d'Europe du sud.

\subsection{Le genre conditionne les devenirs sociaux}

Davantage que toute autre variable, le sexe conditionne et différencie les devenirs sociaux des sortants sans diplôme. Les filles sont les premières à pâtir du chômage : 37 mois en moyenne sur les dix ans contre 23 pour les garçons ; 22 mois pour obtenir un $1^{\text {er }}$ emploi contre 10 pour les garçons; dix ans après leur sortie du système de formation, plus d'une fille non diplômée sur trois est privée d'emploi quand ce n'est le cas que d'un garçon non diplômé sur six. Plus encore, lorsqu'elles sont en emploi, les sortantes sans diplôme sont $27,7 \%$ à occuper un emploi précaire (contre 17,6 \% des garçons), seulement $3 \%$ à être à leur compte (contre 6,3\% des garçons) et surtout, $22,3 \%$, à occuper un emploi stable mais rémunéré en dessous de 1000 euros (contre seulement 2,7 \% des garçons).

Les situations divergent très fortement entre garçons et filles du point de vue des salaires. Parmi les sortants sans diplôme en emploi dix ans après leur sortie du système de formation, les garçons se caractérisent par un salaire net moyen de 1520 euros et un salaire médian de 1430 euros, alors que ces niveaux de salaire ne s'établissent qu'à 1060 euros et 1090 euros chez les filles. Autre manière de présenter ce résultat : parmi les jeunes en emploi dix ans après leur sortie de l'école, une fille non diplômée sur quatre gagne moins de 700 euros, alors que cette situation ne concerne qu'approximativement un garçon sur 43 . 
La principale cause de telles inégalités tient dans le temps de travail : 34,1\% des filles sans diplôme mais en emploi en 2008 travaillent à temps partiel (contre $5 \%$ des garçons) et, parmi elles, $43,4 \%$ travaillent moins de $50 \%$ de la durée légale (ce qui représente $14,8 \%$ des filles non diplômées en emploi, contre seulement 2,8 \% des garçons). Cette fréquence des temps partiels renvoie en partie à la ségrégation des formations et des emplois (Couppié \& Epiphane, 2006) ${ }^{9}$, sans pour autant s'y réduire puisque, dans un secteur comme le commerce (où les femmes non diplômées ne sont d'ailleurs pas surreprésentées par rapport aux garçons non diplômés), les premières sont $45 \%$ à être à temps partiel contre seulement $5 \%$ des seconds.

La population des sans-diplômes apparaît ainsi hautement hétérogène, les difficultés d'insertion professionnelle variant selon un ensemble de variables dont nous avons tenté de quantifier l'influence. Ici comme ailleurs, ce sont le sexe et l'origine sociale qui exercent l'influence la plus grande sur les devenirs sociaux, rappelant que rapports de genre et de classe combinent leur emprise pour produire la structure des inégalités que l'enquête statistique permet de mettre au jour.

\section{L'absence de diplôme : les effets à dix ans d'un signal négatif}

Dans une société combinant un marché de l'emploi fortement concurrentiel et une population de plus en plus diplômée, l'absence de diplôme ne peut manquer d'apparaittre aux employeurs comme un signal négatif (au sens que conferent les économistes à ce terme, c'est-à-dire comme anticipation d'une faible productivité). On peut néanmoins s'interroger sur la pérennité de cet effet de signal ; ce qui est d'ailleurs une manière détournée de prendre la mesure de l'emprise des diplômes sur les devenirs sociaux.

Pour cela, on cherchera, dans cette partie, à comparer les devenirs professionnels des sansdiplômes, dix ans après leur sortie du système de formation, avec ceux des titulaires d'un diplôme de l'enseignement professionnel ${ }^{10}$. Ces diplômes ont pu constituer - dans un autre état des relations entre le système de formation et le système productif - des certifications donnant accès aux fractions supérieures de la classe ouvrière, voire à des positions

9. On pourrait formuler l'hypothèse selon laquelle c'est la concentration des femmes dans des formations et des secteurs faisant un usage intensif des temps partiels qui fait qu'elles sont amenées, beaucoup plus souvent que les hommes, à ne pas travailler à temps plein. La division sexuée des emplois est ainsi très forte parmi les non-diplômés, les filles étant hautement surreprésentées dans le secteur des services $(30,6 \%$ des non-diplômées en emploi travaillent dans ce secteur contre $11,3 \%$ des non-diplômés) et de la santé $(12,4 \%$ contre 1,9\%), alors qu'elles sont sous-représentées dans le secteur de l'industrie et des transports $(14,2 \%$ contre $29,9 \%)$, de la construction $(1,6 \%$ contre $22,7 \%)$ et de l'agriculture $(0,6 \%$ contre 3,5\%). Sur ce point, voir également Couppié, Dupray \& Moulet (2006).

10. Nous nommons ici « enseignement professionnel » les filières qui préparent à des diplômes professionnels de niveau V (CAP-BEP) et IV (" bac pro » et BP). 
relevant de l'encadrement intermédiaire (contremaître) ; or, ils sont aujourd'hui menacés dans ce rôle et servent plutôt de rempart contre le chômage. S'interroger sur la solidité de ce rempart permettra de poser la question des valeurs respectives reconnues aux diplômes et à l'expérience professionnelle, et donc de la possibilité, pour les sans-diplôme, d'accéder à un emploi valorisé malgré leur absence de titre scolaire.

\subsection{Sans-diplômes et diplômés de l'enseignement professionnel sont cantonnés au salariat d'exécution}

S'il est un fait qui caractérise les sortants sans diplôme, et les rapproche des diplômés de niveau CAP-BEP ou même " bac pro ", c'est leur appartenance au salariat d'exécution. Lorsqu'ils sont en emploi, les sortants sans diplôme occupent presque exclusivement des postes d'ouvriers $(53,6 \%$ d'entre eux) et d'employés $(27,4 \%)$. Alors que, pour l'ensemble de la cohorte, $14,4 \%$ des jeunes parviennent à des postes qui relèvent des classes favorisées et $26,3 \%$ des professions intermédiaires, ce n'est le cas que de $1,8 \%$ et $8,6 \%$ des sortants sans diplôme. Pour les diplômés de niveau CAP-BEP, ces taux d'accès aux classes intermédiaires et favorisées s'établissent à des niveaux très proches : 10,3\% d'entre eux parviennent aux professions intermédiaires et seulement $1,4 \%$ aux classes favorisées.

Les jeunes sortis avec un diplôme de niveau « bac » parviennent davantage à s'extraire du salariat subalterne : si seulement 4,1\% d'entre eux parviennent aux positions de "cadres et professions intellectuelles supérieures ", $26 \%$ accèdent aux professions intermédiaires, la majorité occupant des postes d'exécution, d'employés $(31,6 \%)$ ou d'ouvriers (22,5\%), ou se trouvant sans emploi $(10,8 \%)$. Encore doit-on considérer ces derniers chiffres avec circonspection puisque, dans le groupe des bacheliers, coexistent deux fractions dont les devenirs socioprofessionnels sont clairement différenciés. D'un côté, les bacheliers sortis après une classe de " bac pro " sont pour une grande partie d'entre eux ouvriers $(37,1 \%)$ et employés $(25,6 \%)$ dix ans après leur sortie du système de formation; ils connaissent un taux de non-emploi de $8,7 \%$ et n'accèdent guère aux classes favorisées $(1,9 \%)$ ou aux professions intermédiaires $(19 \%)$. De l'autre, les bacheliers sortis après un passage (non diplômant) dans l'enseignement supérieur ne sont ouvriers que pour 14,7\% d'entre eux ${ }^{11}$. Majoritairement employés $(32,7 \%)$, ils ont accès, beaucoup plus que les bacheliers

11. L'enquête à 10 ans du Céreq, auprès de la "Génération 1998 ", ne permet pas de distinguer - parmi les jeunes dont le plus haut diplôme obtenu est un baccalauréat - les bacheliers généraux, technologiques et professionnels. Pour approcher la différence entre ces types de baccalauréat, qui produit des inégalités très sensibles d'insertion professionnelle, on a croisé la variable de plus haut diplôme avec celle qui informe de la dernière classe fréquentée. Si l'on se fie aux données utilisées par S. Lemaire (2005), étant donné que la quasi-totalité des bacheliers généraux $(98,2 \%)$ et la grande majorité des bacheliers technologiques $(90,8 \%)$ poursuivent leurs cursus dans l'enseignement supérieur, contrairement aux « bac pro » (seulement 44,3\%), qui sont nombreux à aller travailler après avoir passé l'examen, la distinction entre bacheliers ayant poursuivi dans le supérieur et bacheliers ayant arrêté leur formation après la terminale recouvre en partie la distinction entre bacheliers généraux et technologiques d'un côté, et bacheliers professionnels de l'autre. 
professionnels, aux professions intermédiaires (32\%) et, plus marginalement, aux catégories supérieures (5,6\%) ; en revanche, ils ont un taux de non-emploi de 11,5\%.

Quant au secteur d'activité, on observe également une convergence entre les sortants sans diplôme et les diplômés de l'enseignement professionnel court, ces deux catégories de jeunes étant surreprésentés dans les mêmes secteurs : l'industrie, les transports, le BTP (bâtiment et travaux publics), le commerce et les services. À l'inverse, ils apparaissent sous-représentés dans le tertiaire administratif, la santé et l'éducation. En termes de positions hiérarchiques au sein du système productif, la coupure s'opère moins entre diplômés et non-diplômés qu'entre, d'un côté, les diplômés de l'enseignement professionnel ainsi que les sortants sans diplôme (mais souvent passés par les filières professionnelles) et, de l'autre, les diplômés de l'enseignement supérieur (court ou long), ou même ceux qui sont passés par l'enseignement supérieur sans décrocher de diplôme.

Un modèle de régression logistique permet de préciser ces constats. Il confirme que, toutes les variables inclues dans le modèle étant tenues constantes, la situation socioprofessionnelle à dix ans des sortants sans diplôme se rapproche de celle des diplômés de niveau CAP-BEP.

Exclus ou presque des emplois stables rémunérés à plus de 1500 euros, les sans-diplômes ont 41,7 fois plus de chances que les diplômés de niveau «bac +3 et plus » d'occuper un emploi d'exécution (ouvrier ou employé), l'odds-ratio s'établissant à 35,6 pour les titulaires d'un CAP ou d'un BEP. Les diplômés de niveau bac occupent une position intermédiaire ; en effet, leurs chances d'échapper aux emplois d'exécution, dix ans après leur sortie du système éducatif, sont plus fortes que celles des catégories précédemment citées mais demeurent faibles au regard de la situation des diplômés de niveau « bac $+2{ }^{12}$. On comprend ainsi la tentation, présente chez une fraction importante des bacheliers professionnels, de poursuivre leurs études dans l'enseignement supérieur - soit en BTS (brevet de technicien supérieur) dans la continuité de leur cursus antérieur (Moullet, 2005), soit dans des voies parfois fort éloignées de leur formation professionnelle. Il s'agit ici de briser ce "plafond de verre " et de retrouver par la bande ce qu'ils nomment parfois la "voie normale» (Beaud, 2002).

Les sans-diplômes n'apparaissent donc pas comme un groupe exclu du monde du travail, ni d'ailleurs comme une "nouvelle classe sociale" (Amossé et Chardon, 2006), même s'ils occupent fréquemment les segments fragilisés d'un salariat d'exécution lui-même fragmenté, pâtissant plus que toute autre catégorie des soubresauts de la conjoncture économique.

12. Au demeurant, si on introduit dans un modèle identique une variable plus précise du niveau d'études en distinguant, parmi les bacheliers, les sortants de " bac pro » des jeunes passés par l'enseignement supérieur, l'odds-ratio s'établit à des niveaux sensiblement différents pour ces deux populations : les premiers ont ainsi 16,8 fois plus de chances d'occuper un emploi d'exécution que les diplômés de niveau "bac + 3 et plus ", contre 10,9 pour les seconds. Preuve là encore que les diplômes professionnels conservent pour fonction essentielle de produire des salariés d'exécution. 


\section{Tableau 2}

Régression logistique de la probabilité...

\begin{tabular}{|c|c|c|c|}
\hline \multirow{2}{*}{\multicolumn{2}{|c|}{ Variables }} & $\begin{array}{l}\text {... d'occuper un emploi } \\
\text { stable rémunéré à } 1500 \\
\text { euros et plus, plutôt que } \\
\text { tout autre emploi } \\
\text { (n=9675) }\end{array}$ & $\begin{array}{l}\text {... d'occuper un emploi } \\
\text { d'exécution, plutôt que } \\
\text { tout autre emploi (parmi } \\
\text { les jeunes qui ont un } \\
\text { emploi) } \\
(n=8896)\end{array}$ \\
\hline & & $\begin{array}{l}\text { Paramètre estimé } \\
\text { (odds-ratio) (1) }\end{array}$ & $\begin{array}{c}\text { Paramètre estimé } \\
\text { (odds-ratio) }\end{array}$ \\
\hline \multicolumn{2}{|l|}{ Constante } & 2,5 & 0,1 \\
\hline $\begin{array}{l}\text { Sexe } \\
\text { Filles (ref.) }\end{array}$ & Garçons & 3,9 & 0,7 \\
\hline $\begin{array}{l}\text { PCS (2) du père } \\
\text { Cadres et professions intellectuelles } \\
\text { supérieures (ref.) }\end{array}$ & $\begin{array}{l}\text { Agriculteurs } \\
\text { Artisans, commerçants, patrons } \\
\text { Professions intermédiaires } \\
\text { Employés } \\
\text { Ouvriers }\end{array}$ & $\begin{array}{c}0,7 \\
\text { ns } \\
\text { ns } \\
0,9^{*} \\
0,7\end{array}$ & $\begin{array}{c}\text { ns } \\
\mathbf{1 , 2 *} \\
1,5 \\
1,6 \\
1,9\end{array}$ \\
\hline $\begin{array}{l}\text { Situation dans l'emploi des deux } \\
\text { parents } \\
\text { Deux parents en emploi (ref.) }\end{array}$ & $\begin{array}{l}1 \text { parent en emploi, l'autre au chômage } \\
\text { Deux parents au chômage } \\
\text { Autre situation }\end{array}$ & $\begin{array}{c}0,9^{*} \\
\text { ns } \\
\text { ns }\end{array}$ & $\begin{array}{l}1,2 \\
\text { ns } \\
\text { ns }\end{array}$ \\
\hline $\begin{array}{l}\text { Zone d'habitation } \\
\text { Pôle urbain (ref.) }\end{array}$ & $\begin{array}{l}\text { Couronne périurbaine } \\
\text { Commune multi-polarisée } \\
\text { Commune rurale }\end{array}$ & $\begin{array}{l}0,8 \\
1,3 \\
0,7\end{array}$ & $\begin{array}{c}1,3 \\
1,2^{*} \\
1,5\end{array}$ \\
\hline $\begin{array}{l}\text { Pays de naissance du père } \\
\text { France (ref.) }\end{array}$ & $\begin{array}{l}\text { Europe du sud } \\
\text { Turquie } \\
\text { Maghreb } \\
\text { Afrique subsaharienne } \\
\text { Asie } \\
\text { Autre }\end{array}$ & $\begin{array}{l}\text { ns } \\
\text { ns } \\
\text { ns } \\
\text { ns } \\
\text { ns } \\
\text { ns }\end{array}$ & $\begin{array}{l}\text { ns } \\
0,4 \\
\text { ns } \\
\text { ns } \\
\text { ns } \\
\text { ns }\end{array}$ \\
\hline $\begin{array}{l}\text { Plus haut diplôme obtenu } \\
\text { Bac }+3 \text { et plus (ref.) }\end{array}$ & $\begin{array}{l}\mathrm{Bac}+2 \\
\text { Bac } \\
\text { CAP-BEP (3) } \\
\text { Sans diplôme }\end{array}$ & $\begin{array}{l}0,7 \\
0,2 \\
0,1 \\
0,1\end{array}$ & $\begin{array}{l}2,3 \\
12,8 \\
35,7 \\
41,7\end{array}$ \\
\hline R² de Nagelkerke (4) & & 0,3 & 0,44 \\
\hline
\end{tabular}

Source : Enquête à 10 ans auprès de la "Génération 1998 », Céreq.

Les chiffres sont significatifs à $5 \%$ sauf ceux repérés par * qui sont significatifs au seuil de $10 \%$; « ns » correspond aux chiffres non significatifs à $5 \%$.

Note de lecture : parmi les jeunes sortis du système de formation en 1998, les garçons avaient 3,9 fois plus de chances que les filles, toutes choses égales par ailleurs, d'occuper un emploi stable rémunéré à hauteur d'au moins 1500 euros plutôt que tout autre emploi.

(1) : L'odds-ratio désigne un rapport de chances. Lorsqu'il est inférieur à 1, il suffit de calculer l'inverse (1/odds-ratio) pour le rendre lisible. Dans le tableau ci-dessus, on dira alors que les jeunes sans diplôme ont 10 fois moins de chances $(1 / 0,1)$, toutes choses égales par ailleurs, d'occuper un emploi rémunéré à plus de 1500 euros et plus, plutôt que tout autre emploi.

(2) : Profession et catégorie sociale.

(3) : Respectivement certificat d'aptitude professionnelle et brevet d'études professionnelles.

(4) : Le $R^{2}$ de Nagelkerke est un coefficient ajusté de détermination généralisé du modèle, qui permet d'en estimer le pouvoir explicatif, c'est-à-dire la part de la variance expliquée. Le premier modèle de régression logistique présenté ci-dessus permettrait donc d'expliquer $30 \%$ de la variance (le $\mathrm{R}^{2}$ sétablissant ici à 0,3). 


\subsection{Parvenus à l'emploi, les sans-diplômes pâtissent peu de leur déficit en capital scolaire}

Des différences minimes se dessinent toutefois au sein du groupe des jeunes qui ne sont pas passés par l'enseignement supérieur : quand ils sont ouvriers, les sans-diplômes occupent moins souvent des postes d'ouvriers qualifiés (56,2\%) ou, quand ils sont employés, des positions d'employés qualifiés ${ }^{13}$ (58,5\%) que les diplômés de niveau CAP-BEP (respectivement $64,8 \%$ et $64,1 \%)$ ou les titulaires du « bac pro » $(64,1 \%$ et $61,7 \%)$. De même, ils sont plus nombreux à occuper des emplois précaires (26\% contre 20,1\% et 16,4\%), à temps partiel $(14,4 \%$ contre $12,5 \%$ et $10,1 \%)$, avec des salaires plus souvent inférieurs à 1500 euros $(64,8 \%$ contre $63,1 \%$ et $55,6 \%)$.

Néanmoins, dix ans après leur sortie de l'École, les écarts entre diplômés de l'enseignement professionnel et sortants sans diplôme sont faibles. Tout se passe comme si, parvenant à obtenir un emploi, les sans-diplômes pâtissaient peu de leur déficit de capital scolaire ; à l'inverse, le pouvoir des diplômes professionnels - particulièrement CAP et BEP - semble se résumer à une protection contre le chômage, dans la mesure où ils ne garantissent pas l'accès aux fractions qualifiées du salariat d'exécution (OQ et EQ), et ne permettent guère une promotion socioprofessionnelle (accès aux professions intermédiaires, du moins dans la première décennie de vie active).

En effet, là où les diplômés de niveau 5 (CAP-BEP) doivent en moyenne patienter moins de 6 mois pour obtenir leur premier emploi, ce temps d'attente moyen s'établit à plus de 9 mois pour les sans-diplômes sortis du système de formation après une $2^{\text {ème }}$ année de CAP-BEP, et à presque un an pour ceux qui ont arrêté pendant ou à la fin de leur $1^{\text {ère }}$ année de CAP-BEP. Malgré ce différentiel sensible, les inégalités de salaire s'avèrent très faibles, voire inexistantes entre ces trois catégories, y compris en 2001 (c'est-à-dire trois ans seulement après leur sortie du système de formation) ; date à laquelle les jeunes sortis sans diplôme après une $2^{\text {ème }}$ année de CAP-BEP obtiennent même, en moyenne, un salaire légèrement supérieur à celui des titulaires d'un diplôme de niveau 5 .

Cela tend à conforter l'idée selon laquelle les employeurs usent du diplôme comme d'un " signal " au moment du recrutement (par économie de temps consacré à la recherche d'information fiable sur les candidats au poste), mais émettent ensuite des jugements fondés davantage sur leur productivité, assiduité, discipline, etc. Il faut rappeler que les individus qui font l'objet de l'enquête à 10 ans auprès de la "Génération 1998 " se sont insérés dans une période d'embellie économique, associant une croissance soutenue à une baisse du chômage. La file d'attente se rétrécissant, les sortants sans diplôme ont accédé à

13. On a repris ici pour l'essentiel la distinction entre employés qualifiés et employés non qualifiés proposée par Chenu et Burnod (2001). 


\section{Graphique 4 \\ Évolution des taux d'emploi des sortants sans diplôme et des titulaires d'un diplôme de niveau 4}

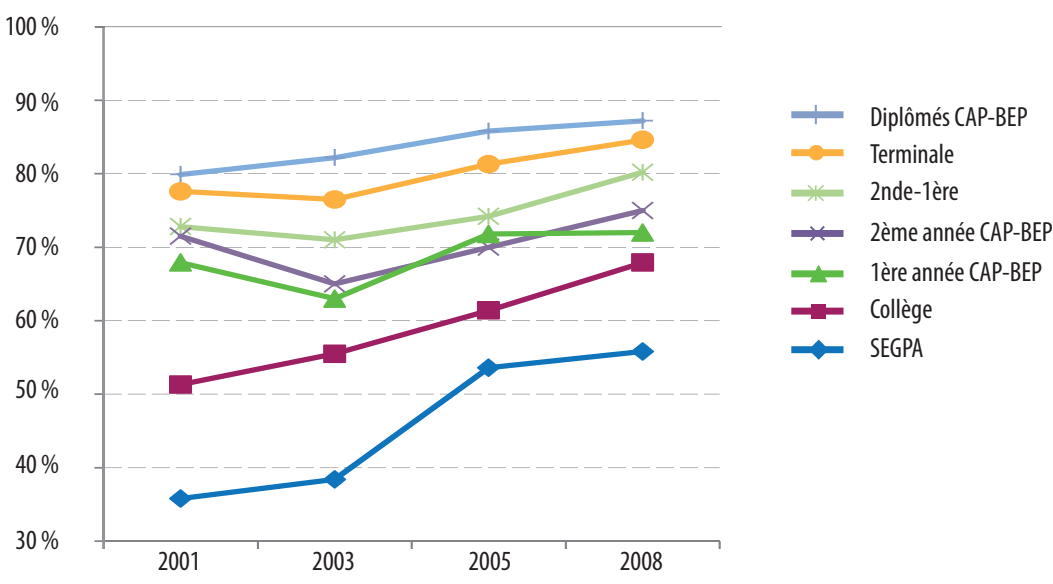

Source : Enquête à 10 ans, auprès de la " Génération 1998 ", Céreq.

Note de lecture : parmi les sortants du système de formation en 1998, les diplômés de niveau CAP étaient 79,9\% à être en emploi en 2001, $82,2 \%$ en $2003,85,5 \%$ en 2005 et $87,2 \%$ en 2008.

Sigles : CAP : certificat d'aptitude professionnelle ; BEP : diplôme d'études professionnelles ; SEGPA : sections d'enseignement général et professionnel adaptées.

des emplois qui leur ont permis d'acquérir une expérience professionnelle et de compenser ainsi le signal négatif que constituait leur absence de titre scolaire ${ }^{14}$.

Cependant, il importe de noter que, contrairement aux titulaires d'un diplôme de niveau 5 , qui voient leur taux d'emploi progresser régulièrement entre les différentes interrogations (passant de 79,9 \% en 2001 à 87,2 \% en 2008), ce taux apparaît plus fluctuant c'est-à-dire plus sensible à la conjoncture économique - pour les sans-diplômes passés par une formation de niveau CAP-BEP.

On peut faire l'hypothèse qu'une partie des jeunes sortis sans diplôme en 1998 avaient alors profité de la période de croissance économique pour saisir une opportunité d'emploi, en décidant par exemple d'écourter un apprentissage ou une formation en lycée professionnel de niveau CAP-BEP. L'absence de diplôme, moins déterminante au moment de leur sortie du système de formation, est devenue un handicap plus sérieux trois ans plus tard dans un contexte de concurrence accrue pour les emplois d'ouvriers et d'employés, et ils ont ainsi été piégés par le retournement de conjoncture de 2001.

14. Plusieurs publications du Céreq (Marchal et al., 2004 ; Joseph et al., 2008) mettent en exergue cet effet de la conjoncture pour les jeunes sortant du système de formation en 2001 et 2004. 
Dès lors, l'expérience professionnelle est-elle susceptible de compenser l'absence de titre scolaire? Si l'on formule les mêmes modèles de régression logistique que précédemment, mais en contrôlant l'expérience professionnelle (à travers une variable indiquant le nombre de mois passés en emploi durant les dix années postérieures à la sortie du système éducatif), les écarts entre non-diplômés et diplômés de niveau 5 (CAP-BEP) s'en trouvent réduits sensiblement ; et ce aussi bien quant à la probabilité d'être en emploi ou non en 2008, d'occuper un emploi d'exécution plutôt que tout autre emploi, ou d'occuper un emploi stable à plus de 1500 euros mensuels plutôt que tout autre emploi. En d'autres termes, à expérience professionnelle identique, sortants sans-diplôme et diplômés de niveau CAP-BEP connaissent des devenirs socioprofessionnels très proches, tout le problème consistant, pour les non-diplômés, à obtenir cette expérience professionnelle !

\section{Conclusion}

Pour conclure, on peut reprendre certains constats en dressant des perspectives de recherche. Tout d'abord, le handicap que constitue l'absence de diplôme n'est pas homogène : ses effets sont modulés par une série de variables qui conditionne la qualité de l'insertion professionnelle. Quatre facteurs de différenciation se détachent : le sexe, la classe de sortie, l'origine sociale et l'origine migratoire. Seules des études qualitatives attentives aux modalités concrètes d'entrée dans la vie - et au rôle que peuvent notamment jouer les discriminations ou le capital social - pourront permettre de comprendre la production concrète de ces inégalités.

Une réflexion s'impose par ailleurs sur les formes de classification et de catégorisation des sortants du système de formation telles qu'elles sont couramment employées dans - et donc consolidées par - les nomenclatures statistiques. Dans la mesure où les écarts sont très faibles, voire nuls, entre les jeunes qui ont arrêté en cours de formation et ceux qui sont allés jusqu'à son terme sans obtenir le diplôme (entre sortants de $1^{\text {ère }}$ et de $2^{\text {ème }}$ année de CAP-BEP par exemple), la traditionnelle distinction entre qualifiés et non-qualifiés apparaît peu pertinente. Sur le marché du travail, c'est bien la possession d'un titre scolaire qui crée des différences plutôt que le fait d'être allé, ou non, au bout de la formation.

Ensuite, les sortants sans diplôme - en particulier ceux qui ont fréquenté les filières professionnelles - apparaissent très proches des diplômés de niveau 5 (CAP-BEP) quant au type d'emplois occupés, aux statuts d'emploi et aux rémunérations ; cela signale la dévalorisation des diplômes délivrés par l'enseignement professionnel court depuis les années 1970. Celui-ci semble avoir aujourd'hui moins pour fonction de former des travailleurs disposant d'une expertise leur permettant d'accéder à des postes d'ouvriers ou d'employés qualifiés, voire à l'encadrement intermédiaire, que de fournir une protection minimale contre le risque de chômage, l'obtention du diplôme constituant, aux yeux des employeurs ou de leurs services de recrutement, un signal de sérieux sur un marché du travail haute- 
ment concurrentiel. S’ils parviennent à franchir la barrière de l'accès à l'emploi, les nondiplômés voient leur situation se rapprocher de celle des diplômés de niveau CAP-BEP.

Enfin, cet article s'inscrit dans la discussion plus générale relative à l'emprise croissante mais paradoxale des diplômes dans la société française. Les titres scolaires s'avèrent en effet de plus en plus nécessaires mais de moins en moins suffisants, notamment pour garantir l'accès à des emplois stables et correctement rémunérés. Encore faut-il nuancer ce constat en distinguant les diplômes délivrés par l'enseignement professionnel court, ceux auxquels une grande partie des jeunes d'origine populaire ont accès et qui ne permettent guère d'entrevoir une sortie du salariat d'exécution, et les diplômes de l'enseignement supérieur, général ou technologique qui - contrairement à certains discours alarmistes faisant des universités des " usines à chômeurs " ${ }^{15}$ - continuent d'ouvrir des perspectives de promotion socioprofessionnelle.

\section{Bibliographie}

Amossé T. \& Chardon O. (2006), « Les travailleurs non qualifiés : une nouvelle classe sociale ", Economie et Statistique, n 393-394, pp. 203-229.

Baudelot C., Benoliel R., Cukrowicz H. \& Establet R. (1981), Les étudiants, l'emploi et la crise, Paris, Maspéro.

Baudelot C. \& Establet R. (2000), Avoir trente ans en 1968 et en 1998, Paris, Seuil.

BeAud S. (2003 [2002]), $80 \%$ au bac... et après? Les enfants de la démocratisation scolaire, Paris, La Découverte.

BEAud S. (2002), "Le rêve de retrouver la "voie normale” : les bacs pro à l'université ", in Gilles Moreau (coord.), Les patrons, l'État et la formation des jeunes, Paris, La Dispute.

Brinbaum Y. \& Kieffer A. (2009), "Les scolarités des enfants d'immigrés de la sixième au baccalauréat : différenciation et polarisation des parcours ", Population, vol. 64, $n^{\circ} 3$, pp. 561-610.

Burnod G. \& Chenu A. (2001), «Employés qualifiés et non qualifiés : une proposition d'aménagement de la nomenclature des catégories socioprofessionnelles ", Travail et Emploi, $\mathrm{n}^{\circ}$ 86, pp. 87-105.

Chauvel L. (1998), " La seconde explosion scolaire : diffusion des diplômes, structure sociale et valeur des titres ", Revue de l'OFCE, n 66, pp. 5-36.

15. Baudelot et al. (1981) avaient en leur temps mis en évidence la fonction conservatrice d'un tel misérabilisme. 
Couppié T. \& Epiphane D. (2006), « La ségrégation des hommes et des femmes dans les métiers : entre héritage scolaire et construction sur le marché du travail », Formation Emploi, vol. 1, n 93, pp. 11-27.

Couppié T., Dupray A. \& Moullet S. (2006), "Les salaires des hommes et des femmes en début de vie active : des sources de disparité variables selon les professions ", Formation Emploi, vol. 1, n 93, pp. 29-47.

Eymard-Duvernay F. \& Marchal E. (1997), Le jugement des compétences sur le marché du travail. Façons de recruter, Paris, Métailié.

Fournier I. \& Silberman R. (2006), « Les secondes générations sur le marché du travail en France : une pénalité ethnique ancrée dans le temps. Contribution à la théorie de l'assimilation segmentée ", Revue française de sociologie, vol. 47, n² 2, pp. 243-292.

Frickey A., Murdoch J. \& Primon J.-L. (2004), Les débuts dans la vie active des jeunes issus de l'immigration après des études supérieures, NEF, $n^{\circ} 9$, Céreq.

GÉHIN J.-P. (2009), « Les politiques de formation professionnelle en région : un processus lent et complexe de construction de l'action publique régionale ", Recherche en éducation, 7 , juin.

GÉHIN J.-P. \& AurAs E. (dir.) (2011), La VAE à l'université : une approche monographique, Rennes, PUR.

Glasman D. \& CEuvrard F. (dir.) (2004), La déscolarisation, Paris, La Dispute.

Gorgeu A. \& Mathieu R. (2009), « La place des diplômes dans la carrière des ouvriers de la filière automobile ", Formation Emploi, n 105, pp. 37-50.

Joseph O., Lopez A. \& Ryk F. (2008), « Génération 2004, des jeunes pénalisés par la conjoncture ", Bref-Céreq, janvier, n 248.

Lainé F. \& Оква M. (2005), «Jeunes de parents immigrés : de l'école au métier », Travail et Emploi, $\mathrm{n}^{\circ} 103$, pp. 79-93.

Lemaire S. (2004-2005), "Que deviennent les bacheliers après leur baccalauréat ?", France, portrait social, INSEE.

Marchal N., Molinari-Perrier M. \& Sigot J.-C. (2004), «Génération 2001. S’insérer lorsque la conjoncture se dégrade ", Bref-Céreq, décembre, $\mathrm{n}^{\circ} 214$.

Meurs D., Pailhé A. \& Simon P. (2006), « Mobilité entre générations d'immigration et persistance des inégalités : l'accès à l'emploi des immigrés et de leurs descendants en France ", Population, n 5-6, pp. 763-801.

Millet M. \& Thin D. (2003), "Une déscolarisation encadrée. Le traitement institutionnel du "désordre scolaire" dans les dispositifs-relais ", Actes de la recherche en sciences sociales, $\mathrm{n}^{\circ} 149$, p. 32-41. 
Millet M. \& Thin D. (2005), Les ruptures scolaires. L'école à l'épreuve de la question sociale, Paris, PUF.

Moullet S. (2005), «Après le bac professionnel ou technologique : la poursuite d'études jusqu’à bac +2 et sa rentabilité salariale en début de vie active ", Économie et Statistique, $\mathrm{n}^{\circ}$ 388-389, pp. 15-36.

Neyrat F. (dir.) (2007), La validation des acquis de l'expérience, Bellecombe-en-Bauges, Le Croquant.

Palheta U. (2011), " Le paradoxe de Willis. Les destins scolaires des jeunes d'origine populaire dans l'Ecole massifiée ", Revue Agone, n 46.

Palheta U. (à paraître 2012), La domination scolaire. Sociologie de l'enseignement professionnel et de son public, Paris, PUF, « Le lien social».

Paugam S. (1991), La disqualification sociale, Paris, PUF.

Rose J. \& Tessier J. (2006), « La certification, nouvel instrument de la relation formationemploi. Un enjeu français et européen ", Relief-Céreq, n 16, juillet.

SPEnce M. (1973), "Job Market Signalling", The Quarterly Journal of Economics, vol. 87, $\mathrm{n}^{\circ} 3$, pp. 355-374.

Verdier E. (2001), "La France a-t-elle changé de régime d'éducation et de formation?", Formation Emploi, $\mathrm{n}^{\circ} 76$.

ZAFFrAN J. (2010), «Entrer en Segpa et en sortir ou la question des inégalités transposées », Formation Emploi, $\mathrm{n}^{\circ} 109$. 